\title{
Investigation into the expression levels of MAGEA6 in esophageal squamous cell carcinoma and esophageal adenocarcinoma tissues
}

\author{
JUN HAO $^{1 *}$, SHUYING LI $^{2 *}$, JINTAO LI $^{1}$, ZHU JIANG $^{1}$, MALIHA GHAFFAR ${ }^{1}$, \\ MINGLIAN WANG ${ }^{1}$, RUNQING JIA ${ }^{1}$, SU CHEN ${ }^{3}$, YANGJUNQI WANG ${ }^{1}$ and YI ZENG ${ }^{1,4}$ \\ ${ }^{1}$ Beijing Key Laboratory of Environmental and Viral Oncology, College of Life Science and Bio-Engineering, \\ Beijing University of Technology, Beijing 100124; ${ }^{2}$ Hebei Key Laboratory for Chronic Diseases, Tangshan \\ Key Laboratory for Preclinical and Basic Research on Chronic Diseases, North China University of Science and Technology, \\ Tangshan, Hebei 063000; ${ }^{3}$ Hubei Key Laboratory of Medical Information Analysis and Tumor Diagnosis and Treatment, \\ Wuhan, Hubei 430074; ${ }^{4}$ Chinese Center for Disease Control and Prevention, National Institute for \\ Viral Disease Control and Prevention, and State Key Laboratory for Infectious Disease Prevention \\ and Control, Beijing 100052, P.R.China
}

Received January 11, 2018; Accepted March 1, 2019

DOI: $10.3892 /$ etm.2019.7735

\begin{abstract}
Esophageal carcinoma (EC) is the sixth most deadly of all cancers. It is among the most malignant cancers due to its highly aggressive nature and low survival rate. The incidence of EC is high in Asia, particularly in Southern areas including China, Iran and Japan. There is a large body of evidence to suggest an association between the melanoma antigen gene (MAGE) family and the initiation of cancer; however, there is no clear evidence to suggest an association between EC and MAGE. Discovery of the chemical and physiological processes relevant to the occurrence of EC is vital for clinicians to diagnose and treat this highly aggressive cancer. The present study focused on the association of EC with the expression of MAGE family member A6 (MAGEA6) at the mRNA and protein levels using gene chip, reverse transcription-quantitative polymerase chain reaction (RT-qPCR)
\end{abstract}

Correspondence to: Dr Jintao Li, Beijing Key Laboratory of Environmental and Viral Oncology, College of Life Science and Bio-Engineering, Beijing University of Technology, 100 Pingleyuan, Chaoyang, Beijing 100124, P.R. China

E-mail: ljt2008@bjut.edu.cn

Professor Shuying Li, Hebei Key Laboratory for Chronic Diseases, Tangshan Key Laboratory for Preclinical and Basic Research on Chronic Diseases, North China University of Science and Technology, 1 Bohai Sea Roadm Caofeidian Area, Tangshan Bay Eco-city, Hebei 063000, P.R. China

E-mail: 1sy5001@sina.com

*Contributed equally

Key words: esophageal neoplasms, adenocarcinoma cell, squamous cell, MAGEA6 protein, malignant transformation, immunohistochemistry and immunohistochemistry. The expression of MAGEA6 in human esophageal squamous cell carcinoma (ESCC) and esophageal adenocarcinoma (EAC) tissue samples were compared with those in paracancerous tissue. The result of the gene chip assay revealed that as the generation grew, there was a significant increase in MAGEA6 transcription in the esophageal epithelial cell line, SHEE Different ESC cell lines also exhibited a significantly higher transcription of MAGEA6 compared with the HaCaT cell line, as determined via reverse transcription-quantitative PCR. An higher positive rate of MAGEA6 expression in ESCC and EAC tissues was also revealed when compared with paracancerous tissues, as determined via immunohistochemistry. The results indicated that MAGEA6 is highly transcribed and expressed in the development of EC and may therefore serve as a novel biomarker for the diagnosis or treatment of EC.

\section{Introduction}

Esophageal carcinoma (EC) has the sixth highest lethality rate among cancers and is the eighth most common cancer worldwide (1). Developing countries in Asia, including China, Turkey and Iran, have the highest incidence rates of EC, where patients are mostly diagnosed with EC in the middle and late stages of the disease, with $15-25 \%$ survival after 5 years $(2,3)$. However, early diagnosis, via highly developed endoscopic techniques, may increase survival rates by enabling the use of early treatment regimens and mitigation techniques $(3,4)$.

The root cause of EC remains unclear; however, environment, sex, race and personal characteristics, including alcohol intake, smoking, viruses and genetic factors have all been linked to its initiation and progression (3). A number of susceptibility genes, such as GSTT1 and CYPIAI $(5,6)$, suppressor genes including $p 53, p 16, A P C, R b$ and $E P B 41 L 3(7,8)$, and oncogenes such as cyclin D1 and EGFR (9), have been shown to serve a role in the development of $\mathrm{EC}$, and have provided significant data regarding the early diagnosis of EC. 
Melanoma antigen gene $(M A G E)$ is a family of tumor-associated antigens that was discovered in malignant tissues by van der Bruggen et al (10) through gene cloning experiments. Research has shown that the $M A G E$ family A (MAGEA), which consists of a group of 12 genes named MAGEA (1-12), is located in humans at the chromosomal location of ChrXq28 $(11,12)$. Expression of MAGE1 has a close association with tumorigenesis, and certain members of the family have been studied as diagnostic markers for various types of carcinoma (12). Previous studies have also shown that the occurrence of EC is a consequence of environmental factors along with human papillomavirus (HPV) infection (13-15). The MAGEA family is highly expressed in different cancer tissues, particularly in ESCC tissues; various cancers in the lung, head and neck, esophagus, bladder, stomach, colorectal, breast, liver and ovary; as well as lymphocytic leukemia $(12,16)$.

Research into the metastasis of esophageal epithelial cells has indicated that MAGEA6 may be important in the occurrence of EC (12). However, the specific association between MAGEA6 and the incidence of EC, as well as its mechanism remain unclear. Therefore, the present study focused on clarifying the biological association between the expression of MAGEA6 throughout different stages of the expression process (mRNA transcription and protein expression) and the occurrence of EC. Furthermore, a detailed analysis of clinically derived EC clinical samples and EC cell lines was conducted.

In the present study, MAGEA6 was detected in clinical EC samples and EC cell lines. Gene chip and reverse transcription-quantitative (RT-q) PCR was designed to reveal the transcription levels of MAGEA6 in different generations of human immortal esophageal epithelial cell lines and in different ESC cell lines, the results of which may indicate whether the transcription of MAGEA6 is higher in EC cells than common human cell lines and if this changes during the development of EC. Immunohistochemistry detection was also designed to indicate the positive rate of MAGEA6 in EC tissue when compared with paracancerous tissue. The results of these experiments should reveal whether there is close association between EC and MAGEA6, and if MAGEA6 could be treated as a novel biomarker of EC.

\section{Materials and methods}

Malignant transformation of human esophageal epithelial cells detected via gene chip analysis. The present study was conducted with approval from the Ethics Committee of North China University of Science and Technology (Tangshan, China). The SHEE immortal esophageal epithelial cell line was obtained by HPV18E6E7 inducement at the Medical College of Shantou University (Shantou, China). A human embryonic esophageal epithelial cell line, obtained from the Medical College of Shantou University, was firstly infected with HPV18 E6E7-AVV and then treated with tumor-promoting factor (12-O-Tetradecanoylphorbol 13-Acetate, TPA) to induce transformation into malignant SHEE cells $(17,18)$. This immortal esophageal epithelial cell line retains the characteristics of monolayer growth, contact inhibition and squamous epithelium origin, and when continually cultivated, readily undergoes malignant transformation (19). The normal esophageal epithelial cells were transformed into esophageal squamous epithelial cells after 55 generations. Changes of $\sim 47,000$ transcriptomes transcription within the cells were detected using gene chips. This involved detection of total RNA using the gene chips (GeneChip Human Genome U133 Plus 2.0; Affymetrix; Thermo Fisher Scientific, Inc., Waltham, MA, USA) via their reverse transcribed cDNA (20). Finally, the transcription levels of MAGE family genes, including MAGEA6, were determined by comparing the $\mathrm{Cq}$ values detected by the gene chips in the 24, 48, 60 and 76th generations of the malignant-transformed esophageal epithelial cells.

Detection of MAGEA6 transcription using reverse transcription-quantitative polymerase chain reaction ( $R T-q P C R)$ Preparation of $c D N A$. Four ESCC cell lines induced from esophageal epithelial cells, namely EC109, EC9706, CEC-3 and KYSE150, were used in the present study. The EC109 and EC9706 cell lines were obtained from the Chinese Academy of Medical Sciences, Peking Union Medical College (Beijing, China). The KYSE150 cell line was obtained from Shanghai Institutes for Biological Sciences, Chinese Academy of Sciences (Shanghai, China). The Chinese Esophageal Cancer-3 (CEC-3) cell line, constructed by Professor Zeng Yi, was obtained from the National Institute for Viral Disease Control and Prevention, Chinese Center for Disease Control and Prevention (Beijing, China). HaCaT cells, also obtained from Professor Yi Zeng, were used as a control. The EC109, EC9706, CEC-3, KYSE150 and HaCaT cells were lysed using TRIzol (cat. no. 15596-026; Invitrogen; Thermo Fisher Scientific, Inc.). Total RNA was extracted from the cells following lysis, dissolved in diethylpyrocarbonate water and then authenticated and measured through agarose gel electrophoresis to ensure the integrity of the RNA. The DNA that was mixed with the RNA during lysis was removed by the addition of RQ1 DNase. The cDNA of all the cell lines was then synthesized using the Reverse Transcription System MK (cat. no. A3500; Promega Corporation, Madison, WI, USA). The temperature protocol was as follows: $42^{\circ} \mathrm{C}$ for $1 \mathrm{~h}, 95^{\circ} \mathrm{C}$ for $5 \mathrm{~min}$ and $4^{\circ} \mathrm{C}$ for $10 \mathrm{~min}$.

RT-qPCR. SYBR-Green reagent (cat. no. 4472908; Applied Biosystems, Thermo Fisher Scientific, Inc.) was used for qPCR analysis [an $8 \mu \mathrm{l}$ system containing $4 \mu \mathrm{l}$ Fast MIX (a ready-to-use cocktail, containing antibody-mediated fast hot start Taq DNA Polymerase, SYBR-Green I fluorescent dye, $\mathrm{MgCl}_{2}$, dNTP Mix and stabilizers), $0.25 \mu \mathrm{l}$ of forward and reverse primers, $1.5 \mu \mathrm{l}$ Nuclease-Free Water and $2 \mu \mathrm{l}$ cDNA (synthesized as aforementioned)] (Table I). The thermocycling conditions were as follows: 40 cycles of denaturation at $95^{\circ} \mathrm{C}$ for $10 \mathrm{~min}$, annealing at $60^{\circ} \mathrm{C}$ for $1 \mathrm{~min}$ and elongation at $72^{\circ} \mathrm{C}$ for $1 \mathrm{~min}$.

Human GADPH and some genes of the MAGE family were screened using RT-qPCR according to the results of the gene chip analysis. The primer sequences used in RT-qPCR (Table II) were designed using Beacon Designer 7.92 (Premier Biosoft International, Palto Alto, CA, USA). Optimally ranked primers were selected, and were synthesized by AuGCT Co., Ltd GADPH served as the internal control. Upon completion of the amplification, the obtained $\mathrm{Cq}$ values were used in the following formulae for analysis: $\Delta \mathrm{Cq}=\mathrm{Cq}$ (target gene) $\mathrm{Cq}$ (internal control); $\Delta \Delta \mathrm{Cq}=\Delta \mathrm{Cq}$ (experimental group) $-\Delta \mathrm{Cq}$ (control group); relative expression of the target gene to the 
Table I. Reverse transcription-quantitative polymerase chain reaction system.

\begin{tabular}{lc}
\hline Component & Volume $(\mu \mathrm{l})$ \\
\hline Fast mix & 4 \\
F Primer & 0.25 \\
R Primer & 0.25 \\
Nuclease-free water & 1.5 \\
cDNA & 2 \\
Total & 8 \\
\hline
\end{tabular}

control $=2^{-\triangle \triangle C q}$ (21). GAPDH was regarded as having an expression level of 1 and target gene expression was calculated relative to that of $G A P D H$.

Detection of MAGEAexpression using immunohistochemistry (IHC). Fresh surgically resected tissue slices from 107 patients (average age, 56 years; age range, 41-77 years; 58 male and 49 female, only tissues from 98 patients were successfully produced into slice samples for IHC detection) were used. The samples included 51 slices of ESCC tissue, 47 slices of EAC tissue and 98 paracancerous tissue slices as the control group, every paracancerous tissues samples was took around the EC tissues we used. The samples were obtained from January 2015 to December 2016 after each specimen donor provided signed consent. All specimen donors were pathologically diagnosed with EC and treated at the Pathology Department of Tangshan People's hospital in Tangshan, Hebei Province. The ESCC and EAC tissue slices ( $2 \mu \mathrm{m}$ thick), together with the paracancerous tissue, slices were fixed with $4 \%$ paraformaldehyde for $24 \mathrm{~h}$ at $25^{\circ} \mathrm{C}$, then sealed with paraffin wax for further use. Sealed slices were subsequently incubated at $56^{\circ} \mathrm{C}$ overnight prior to paraffin wax washing with $100 \%$ xylene twice for $15 \mathrm{~min}$. Subsequent rinsing with 100, 95 and $70 \%$ ethanol for $5 \mathrm{~min}$ respectively was conducted to clean the slices. The cleaned slices were boiled in a pressure cooker with $0.01 \mathrm{M}$ citrate buffer solution 5 times $(2 \mathrm{~min}$ each) to activate and retrieve the antigens on the surface, and then cooled to $25^{\circ} \mathrm{C}$. Afterwards, the slices were immersed in $3 \%$ hydrogen peroxide at $25^{\circ} \mathrm{C}$ for $20 \mathrm{~min}$ to block endogenous peroxidase activity. Rabbit anti-human MAGEA6 polyclonal antibodies (primary antibodies; 1:50; cat. no. 14602-1-AP) from ProteinTech Group, Inc., Chicago, IL, USA were then added and the slices were maintained at $4^{\circ} \mathrm{C}$ overnight. Then, the slices were washed with phosphate-buffered saline (PBS) 4 times (4 min each) and primary antibody enhancer [Boster Biological Technology; Ready-to-use SABC-POD (mouse/rabbit IgG) kit; cat. no. SA1020] was added. The slices were incubated with secondary antibodies and stained for 0.5-1 min with the Dako REAL $^{\mathrm{TM}}$ EnVision $^{\mathrm{TM}}$ Detection System, Peroxidase/DAB+, Rabbit/Mouse (DAB; cat. no. K500711). Then samples were treated with hematoxylin for $30 \mathrm{sec}$ at $25^{\circ} \mathrm{C}$. Following thorough dehydration and soaking in xylene, the stained slices were sealed and observed under a microscope.

The data from the IHC assay were then analyzed by two double-blinded people (Dr Minglian Wang and Miss Zhu Jiang).
Cells with positive MAGEA6 protein staining primarily appeared brown-yellow, and the strength of color indicated the extent of protein expression. Cells with no clear staining were considered negative. The total positive rates for MAGEA6 in the ESCC,EAC and paracancerous tissues were first calculated. The total EC positive rate was also calculated. The sensitivity and specificity of MAGEA6 IHC positive staining for ESCC and EAC tissues compared with paracancerous tissues were then analyzed, and the positive and negative predictive values were calculated in order to determine whether the expression of MAGEA6 has the potential to serve as a marker of ESCC and EAC.

Statistical analysis. The differences in MAGEA6 expression level in the EC cell lines compared with the HaCaT cell line were analyzed with Duncan's multiple range test $(\alpha=0.05)$ using SPSS19 software (IBM Corp., Armonk, NY, USA). P<0.05 was considered to indicate a statistically significant difference.

\section{Results}

Malignant transformation in different generations of human esophageal epithelial cells detected by gene chip analysis. Through gene chip analysis, $\sim 47,000$ transcriptomes of total RNA were detected in the esophageal epithelial cells and specific transcripts were observed to undergo gradual changes as the number of cell generations increased. Among them, a change in MAGEA6 transcription was clearly detected. The results demonstrated that the transcription level of MAGEA6 was markedly increased after the 48th generation from $<8.9$ to $>1,097$ in the 60th generation (Fig. 1). Furthermore, MAGEAl, $M A G E A 3$ and $M A G E B 2$, which were highly transcribed in different types of carcinoma, exhibited almost no transcription in EC109 and EC9706 cell lines.

Detection of MAGEA6 expression at the transcription level in EC cell lines using RT-qPCR. The expression of MAGEA6 mRNA was detected using RT-qPCR in four EC cell lines (EC109, EC9706, CEC-3 and KYSE150) and in the human immortalized epidermal HaCaT cell line, using GAPDH as an internal control. The amplification of target genes was divided into fluorescent background signal, fluorescent signal exponential amplification and platform stages, and the RT-qPCR melting curve signified the high specificity of the primers for MAGEAC and $G A D P H$. These results indicated that the RT-qPCR method used in the present study was reliable (data not shown).

Comparison of the transcription of MAGEA6 and GAPDH in the four esophageal cancer cell lines and $\mathrm{HaCaT}$ cells using Duncan's multiple range test revealed that the $\mathrm{P}$ value between groups was $\mathrm{P}<0.001$, indicating that the transcription level of MAGEA6 (relative to GADPH) in the four esophageal cancer cell lines was significantly higher compared with that in the $\mathrm{HaCaT}$ cell line (Fig. 2).

Detection of MAGEA6 expression in carcinoma and paracancerous tissues using IHC. In the present study, 196 tissue specimens were analyzed using IHC and the results compared. These specimens included 51 specimens of ESCC tissue, 47 specimens of EAC tissue and 98 specimens of paracancerous tissue. 
Table II. Primer sequences for reverse transcription-quantitative polymerase chain reaction.

\begin{tabular}{lcl}
\hline Primer & Direction & Sequence \\
\hline GAPDH & F & \\
MAGEA6 & R & 5'-ACCACAGTCCATGCCATCAC-3' \\
& F & 5'-TCCACCACCCTGTTGCTGTA-3' \\
MAGEA1 & R & 5'-CGGTCACAAAGGCAGAAAT-3' \\
& F & 5'-AGGCAGGTGGCAAAGATG-3' \\
MAGEA3 & R & 5'-CGGCCGAAGGAACCTGACCCAG-3' \\
MAGEB2 & F & 5'-GCTGGAACCCTCACTGGGTTGCC-3' \\
& R & 5'-TGGAGGACCAGAGGCCCCC-3' \\
& F & 5'-GGACGATTATCAGGAGGCCTGC-3' \\
& R & 5'-CTGACTTCCGCTTTGGAGGC-3'
\end{tabular}

MAGE, melanoma antigen gene.

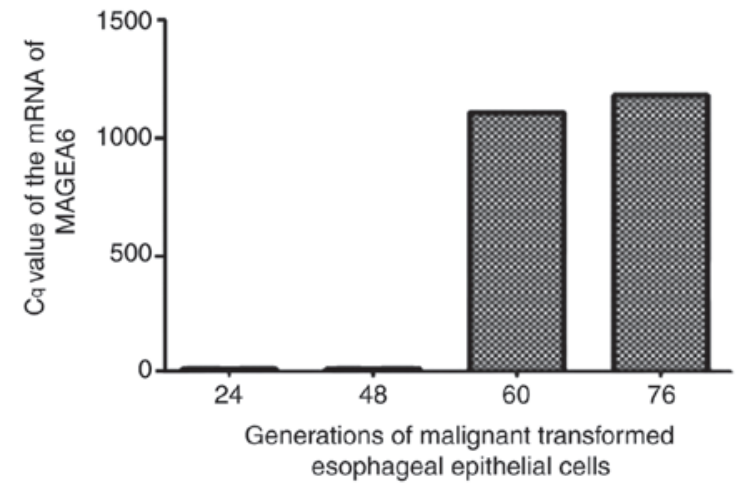

Figure 1. Changes in the transcription level of MAGEA6 gene. Gene expression was analyzed as the generations of a malignantly transformed esophageal epithelial cell line increased, using gene chip analysis. The transcription levels of MAGEA6 underwent the greatest changes from the 24th to the 76th generation; the $\mathrm{Cq}$ value rose markedly from $<8.9$ in the 40th generation to $>1,097$ in the 60 th generation. MAGE, melanoma antigen gene.

Analysis of the ESCC tissues and corresponding paracancerous tissues revealed that 46 ESCC tissues were MAGEA6 positive and 5 were MAGEA6 negative; all 51 samples of paracancerous tissues were MAGEA6 negative. Therefore, when analyzing these two types of tissue, the sensitivity was $90.2 \%$ (46/51), the specificity was $100 \%$ (51/51), the positive predictive value was $100 \%(46 / 46)$ and the negative predictive value was $91.1 \%(51 / 56)$.

Analysis of the EAC tissues and paracancerous tissues revealed that 46 EAC tissue samples were MAGEA6 positive and 1 was MAGEA6 negative; all 47 paracancerous tissue samples were MAGEA6 negative. Therefore, for distinguishing between these two types of tissue, the sensitivity was $97.9 \%$ (46/47), the specificity was $100 \%$ (47/47), the positive predictive value was $100 \%$ (46/46) and the negative predictive value was $97.9 \%$ (47/48). Representative images of IHC staining are presented in Figs. 3-7, the positive and negative rates of MAGEA6 in the tissues as determined by IHC are shown in Table III and the sensitivity, specificity, positive and negative predictive values of MAGEA6 expression analysis using IHC are shown in Table IV.

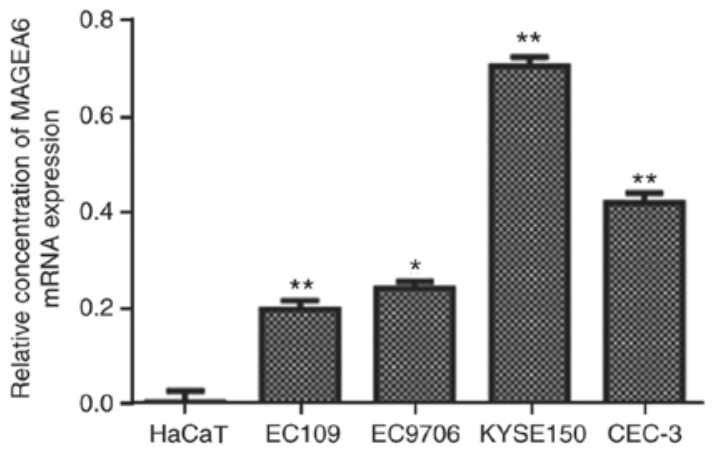

Figure 2. Transcription levels of MAGEA6 in various cell lines detected by reverse transcription-quantitative polymerase chain reaction. In the EC109, EC9706, KYSE150 and CEC-3 esophageal carcinoma cell lines, the expression of MAGEA6 mRNA was significantly higher compared with that in the HaCaT cell line. The expression ratios were as follows: EC109, 118.9/1; EC9706, 179.6/1; KYSE150, 688.3/1; CEC-3, 402.1/1. ${ }^{*} \mathrm{P}<0.05 ;{ }^{* *} \mathrm{P}<0.01$. MAGE, melanoma antigen gene.

\section{Discussion}

The causes of EC are highly complex, and there are limited options for its early diagnosis and treatment $(3,4,22)$. EC is typically diagnosed in the middle and late stages of the disease $(22,23)$, with conventional therapy using radiotherapy, chemotherapy and surgery $(24,25)$. Progress has been made via the detection of a number of susceptibility genes and specific oncogenes $(7,9)$, which provide an important starting point for research into target drug discovery.

The MAGEA family has garnered much interest in the research community for its potential as a target for new therapeutics. Genes belonging to the MAGEA family have been demonstrated to be specifically expressed in numerous types of tumor cells and tissues $(13,26)$; for example, the MAGEA3 is highly expressed in pancreatic cancer (27). Therefore, MAGEA is a significant target in tumor-targeting therapy (13). Although preliminary research has documented an association between the MAGEA family and the occurrence of EC $(28,29)$, the specific relationship remains unclear, with no firm data available to clarify the connection. 


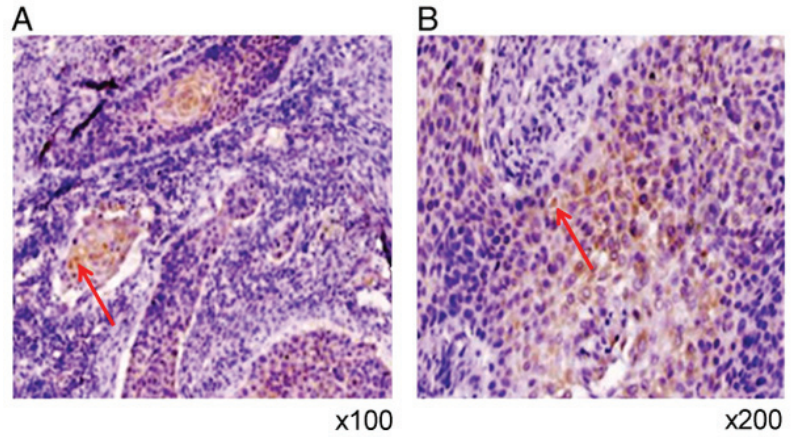

Figure 3. Light microscopy images of the positive immunohistochemical staining of MAGEA6 in esophageal squamous cell carcinoma. Original magnification (A) x100 and (B) x200. MAGEA6, melanoma antigen gene A6. Red arrows indicate positive staining of MAGEA6 protein (Red-yellow in color).
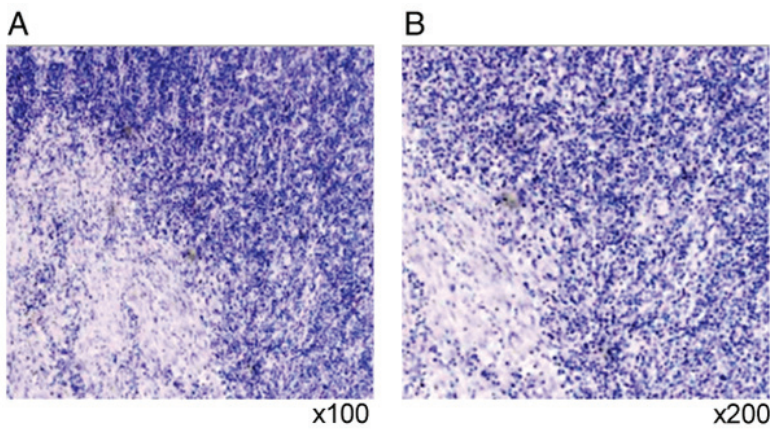

Figure 4. Light microscopy images of the negative immunohistochemical staining of MAGEA6 in esophageal squamous cell carcinoma. Original magnification (A) x100 and (B) x200. Cells were stained for MAGEA6, but appeared negative. MAGEA6, melanoma antigen gene A6.
A

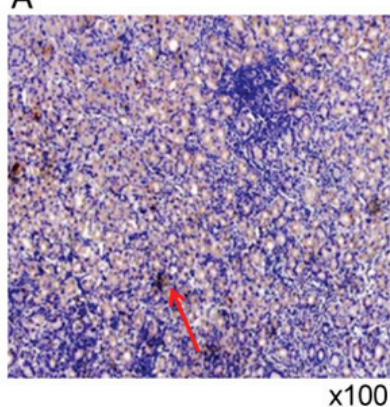

B

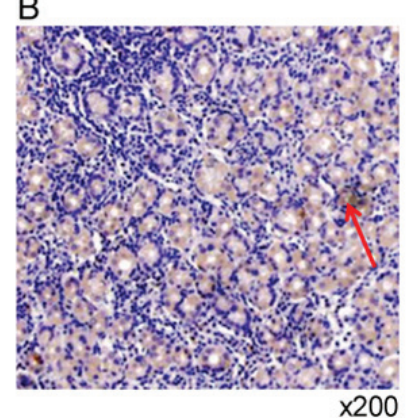

Figure 5. Light microscopy images of the positive immunohistochemical staining of MAGEA6 in esophageal adenocarcinoma. Original magnification (A) x100 and (B) x200. Red arrows indicate positive staining of MAGEA6 protein (Red-yellow in color). MAGEA6, melanoma antigen gene A6.

The current research team has a continued interest in EC, and has previously reported a malignant transformation model for esophageal epithelial cells $(17,18)$. Using this model, changes in gene transcript levels over increasing cell generations were analyzed with a gene chip assay in the present study. The results demonstrated that the transcription level of MAGEA6 rapidly increased as malignant transformation occurred in the esophageal epithelial cells from the 48th generation. From this, it may be speculated that the transcription of MAGEA6
A

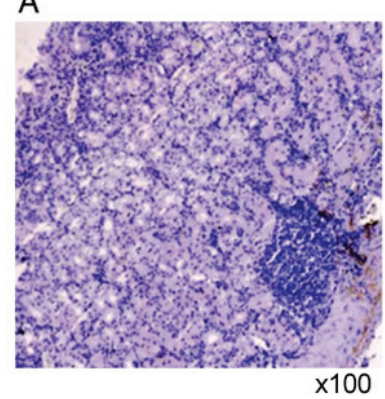

B

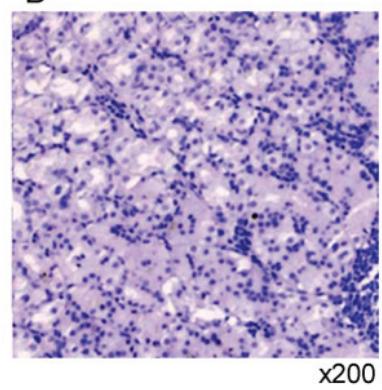

Figure 6. Light microscopy images of the negative immunohistochemical staining of MAGEA6 in esophageal adenocarcinoma. Original magnification (A) x100 and (B) x200. Cells were stained for MAGEA6, but appeared negative. MAGEA6, melanoma antigen gene A6.
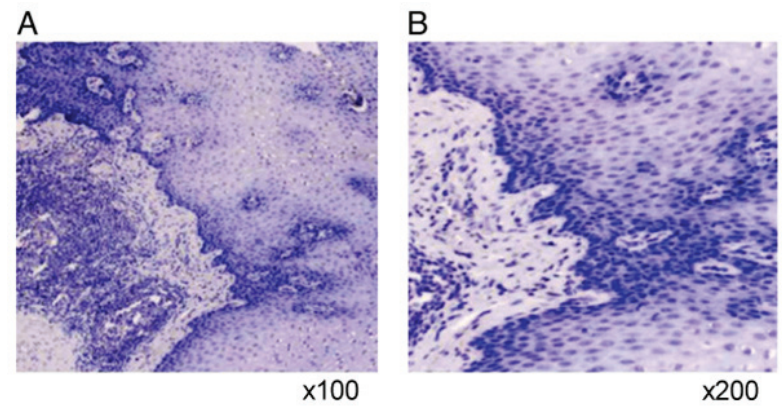

Figure 7. Light microscopy of the negative immunohistochemical staining of MAGEA6 in paracancerous tissue. Original magnification (A) x100 and (B) x200. Cells were stained for MAGEA6, but appeared negative. MAGEA6, melanoma antigen gene A6.

is closely associated with the occurrence of EC. However, since only one set of gene chip detection data was obtained, additional data concerning the transcription of MAGEA6 in different generations of malignantly transformed esophageal epithelial cells are required.

To obtain further evidence of MAGEA6 expression at the mRNA level, RT-qPCR analysis was conducted. The ratio of the transcription level of MAGEA6 in KYSE150 and HaCaT cells was calculated to be 688.3. This cell line exhibited the highest MAGEA6 transcription level among all the carcinoma cell lines that were tested. In the EC109, EC9706 and CEC-3 cell lines, the ratios were 118.9, 179.6 and 402.1, respectively, also indicating much higher transcription levels of MAGEA6 than were observed in the $\mathrm{HaCaT}$ cells. This result demonstrated that MAGEA6 was generally expressed among these most prevalent esophageal cell lines, and provided good evidence for the close association between the expression of MAGEA6 and the occurrence of EC.

To further investigate the association between EC and MAGEA6, specimens of ESCC and EAC tissues were obtained from patients in one of the aforementioned geographical areas with a high prevalence of EC. The MAGEA6 positive rates of the tissues were detected via IHC and, notably, the positive rate differed significantly between the EC tissues and paracancerous tissues. Paracancerous tissues exhibited no expression of MAGEA6 while the total EC tissues presented a very high positive rate of $93.9 \%$. Positive rates were 90.2 and $97.9 \%$ 
Table III. Summary of MAGEA6 immunohistochemical staining results.

\begin{tabular}{lcccc}
\hline Tissues & Positive cases (n) & Negative cases (n) & Positive rate (\%) & Total positive rate (\%) \\
\hline Esophageal squamous cell carcinoma & 46 & 5 & 90.2 & $93.9^{\mathrm{a}}$ \\
Esophageal adenocarcinoma & 46 & 1 & 97.9 & 0 \\
Paracancerous tissues & 0 & 98 & 0 & 0 \\
\hline
\end{tabular}

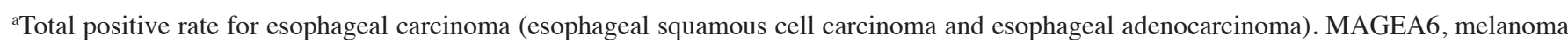
antigen gene A6.

Table IV. Sensitivity, specificity, positive and negative values of MAGEA6 expression.

\begin{tabular}{lcc} 
Parameter & vs. paracancerous tissues $(\%)$ & vs. paracancerous tissues $(\%)$ \\
\hline Sensitivity & 90.2 & 97.8 \\
Specificity & 100 & 100 \\
Positive predictive value & 100 & 100 \\
Negative predictive value & 91.1 & 97.9 \\
\hline
\end{tabular}

MAGEA6, melanoma antigen gene A6.

in the ESCC and EAC tissues, respectively. The sensitivity of the MAGEA6 IHC detection for ESCC and EAC tissues compared with paracancerous tissues was 90.2 and $97.9 \%$, respectively. Furthermore, the specificity for ESCC and EAC tissues compared with paracancerous tissues and the positive predictive values were all $100 \%$. The negative predictive values for ESCC and EAC tissues were also high at 91.1 and $97.9 \%$, respectively. From this analysis, it may be concluded that the sensitivity and specificity were sufficiently high to indicate that MAGEA6 expression at the protein level may be treated as an indicator of the occurrence of ESCC and EAC. This is supported by the high positive and negative predictive values. These results indicate that MAGEA6 is highly expressed in malignantly transformed cells and scarcely expressed in paracancerous tissues.

From the aforementioned results, it may be concluded that the expression of MAGEA6 in EC cells is much higher than that in normal esophageal epidermal cells, and may be easily detected using various methods. These results serve as an important reference to support the use of MAGEA6 as a new drug target for EC treatment, and the expression level may also be an important prognostic index of EC.

In conclusion, the present study demonstrated via gene chip analysis, that the transcription level MAGEA6 in embryonic esophageal epithelial cells increases during carcinogenesis. The greatest increase in the transcription level of MAGEA6 occurred from the 48th to the 60th cell generation, during which the $\mathrm{Cq}$ level of transcription increased from $<8.9$ to $>1,097$. The markedly higher transcription levels identified in all the studied esophageal cell lines strongly indicate the high general level of MAGEA6 expression from the transcription level. The quantity of the MAGEA6 translation product is also much higher in EC tissue than in paracancerous tissues. Therefore, it is concluded that the expression of MAGEA6 has a close association with the occurrence of EC, and MAGEA6 is strongly suggested to be a new oncogene in EC.

\section{Acknowledgements}

The authors would like to thank Mr. Liu Hongwei (Beijing Key Laboratory of Environmental and Viral Oncology, College of Life Science and Bio-Engineering, Beijing University of Technology, Beijing, China) for providing technical support with statistical analysis using SPSS19 software.

\section{Funding}

Funding was provided by the Scientific Research Project of Beijing Educational Committee (KM201910005004), the Beijing Natural Science Foundation (grant no. 5162003), and the Beijing University of Technology Foundation (grant no. 015000514314004).

\section{Availability of data and materials}

The datasets used and analyzed during the current study are available from the corresponding author on reasonable request.

\section{Authors' contributions}

JH wrote the manuscript, designed RT-qPCR, performed the RT-qPCR with the help of ZJ, and took the photos of immunohistochemical analysis. YW performed the gene chip and the RT-qPCR, contributed to the writing of the paper. ZJ and RJ performed immunohistochemical analysis, performed the literature search and designed the RT-qPCR procedure. MW performed immunohistochemical analysis, helped operated RT-qPCR and wrote the manuscript. SL obtained 
patient tissue, performed immunhistochemical analysis and delivered samples to Beijing Key Laboratory of Environmental and Viral Oncology. JL designed the gene chip experiment and completed gene sequencing with help from MG and YW, who collectively analysed the results of gene chip analysis and provided assistance with immunohistochemistry. MG and JL also helped with the writing of manuscript. RJ performed the literature search and wrote the manuscript. SC performed all the statistical analysis. YZ determined how to detect the transcription and expression of MAGEA6 and performed the literature search. MB helped revised some parts of the manuscript.

\section{Ethics approval and consent to participate}

The present study was conducted with approval from the Ethics Committee of North China University of Science and Technology (approval no. 2015119 and 2018216). All participants provided signed consent.

\section{Patient consent for publication}

Not applicable.

\section{Competing interests}

The authors declare that they have no competing interests.

\section{References}

1. Ferlay J, Shin HR, Bray F, Forman D, Mathers C and Parkin DM: Estimates of worldwide burden of cancer in 2008: GLOBOCAN 2008. Int J Cancer 127: 2893-2917, 2010.

2. Zeng Y, Liang W, Liu J, He J, Ng CSH, Liu CC, Petersen RH, Rocco G, D'Amico T, Brunelli A, et al: Esophageal cancer in elderly patients: A population-based study. J Thorac Dis 10: 448-457, 2018.

3. Domper Arnal MJ, Ferrández Arenas Á and Lanas Arbeloa Á: Esophageal cancer: Risk factors, screening and endoscopic treatment in Western and Eastern countries. World J Gastroenterol 21: 7933-7943, 2015

4. Lei J, Tian Y,Zhu SC, Han Q, Wei Y, Yang S and Shi DP: Preliminary study of IVIM-DWI and DCE-MRI in early diagnosis of esophageal cancer. Eur Rev Med Pharmacol Sci 19: 3345-3350, 2015.

5. He F, Liu C, Zhang R, Hao Z, Li Y, Zhang N and Zheng L: Association between the Glutathione-S-transferase T1 null genotype and esophageal cancer susceptibility: A meta-analysis involving 11,163 subjects. Oncotarget 9: 15111-15121, 2018.

6. Zakiullah, Saeed M, Ali S, Javed N, Khisroon M, Muhammad B, Khuda F, Ahmad S and Ismail M: Genetic susceptibility to esophageal cancer due to CYP1A1 gene variant rs4646903 in tobacco addicted patients of Pashtun ethnicity: A case control study in Khyber Pakhtunkhwa province of Pakistan. Asian Pac J Cancer Prev 15: 6715-6720, 2014.

7. Raja S, Godfrey TE and Luketich JD: The role of tumor suppressor genes in esophageal cancer. Minerva Chir 57: 767-780, 2002.

8. Zeng R, Liu Y, Jiang ZJ, Huang JP, Wang Y, Li XF, Xiong WB, Wu XC, Zhang JR, Wang QE and Zheng YF: EPB41L3 is a potential tumor suppressor gene and prognostic indicator in esophageal squamous cell carcinoma. Int J Oncol, 2018.

9. Shibata-Kobayashi S, Yamashita H, Okuma K, Shiraishi K, Igaki H, Ohtomo K and Nakagawa K: Correlation among 16 biological factors [p53, p21(waf1), MIB-1 (Ki-67), p16(INK4A), cyclin D1, E-cadherin, Bcl-2, TNF-alpha, NF-kappaB, TGF-beta, MMP-7, COX-2, EGFR, HER2/neu, ER, and HIF-1alpha] and clinical outcomes following curative chemoradiation therapy in 10 patients with esophageal squamous cell carcinoma. Oncol Lett 5: 903-910, 2013.
10. van der Bruggen $\mathrm{P}$, Traversari $\mathrm{C}$, Chomez $\mathrm{P}$, Lurquin $\mathrm{C}$, De Plaen E, Van den Eynde B, Knuth A and Boon T: A gene encoding an antigen recognized by cytolytic $\mathrm{T}$ lymphocytes on a human melanoma. Science 254: 1643-1647, 1991.

11. Zou C, Shen J, Tang Q, Yang Z, Yin J, Li Z, Xie X, Huang G, Lev D and Wang J: Cancer-testis antigens expressed in osteosarcoma identified by gene microarray correlate with a poor patient prognosis. Cancer 118: 1845-1855, 2012.

12. Lee TB, Lim SC, Moon YS and Choi CH: Melanoma antigen gene family A as a molecular marker of gastric and colorectal cancers. Oncol Rep 30: 234-238, 2013.

13. Yang Y and Fa X: Role of IL-10 gene polymorphisms on the susceptibility for esophageal cancer and its association with environmental factors. Int J Clin Exp Pathol 8: 9580-9585, 2015.

14. Zang B, Huang G, Wang X and Zheng S: HPV-16 E6 promotes cell growth of esophageal cancer via downregulation of miR-125b and activation of Wnt/ $\beta$-catenin signaling pathway. Int J Clin Exp Pathol 8: 13687-13694, 2015.

15. Sarkar J, Dominguez E, Li G, Kusewitt DF and Johnson DG: Modeling gene-environment interactions in oral cavity and esophageal cancers demonstrates a role for the $\mathrm{p} 53 \mathrm{R} 72 \mathrm{P}$ polymorphism in modulating susceptibility. Mol Carcinog 53: 648-658, 2014.

16. Chen YT, Panarelli NC, Piotti KC and Yantiss RK: Cancer-testis antigen expression in digestive tract carcinomas: Frequent expression in esophageal squamous cell carcinoma and its precursor lesions. Cancer Immunol Res 2: 480-486, 2014.

17. Shen ZY, Cen S, Xu LY, Cai WJ, Chen MH, Shen J and Zeng Y: E6/E7 genes of human papilloma virus type 18 induced immortalization of human fetal esophageal epithelium. Oncol Rep 10: 1431-1436, 2003.

18. Shen ZY, Xu LY, Li EM, Cai WJ, Chen MH, Shen J and Zeng Y: Telomere and telomerase in the initial stage of immortalization of esophageal epithelial cell. World J Gastroenterol 8: 357-362, 2002.

19. Shen ZY, Xu LY, Li EM, Cai WJ, Shen J, Chen MH, Cen S, Tsao SW and Zeng Y: The multistage process of carcinogenesis in human esophageal epithelial cells induced by human papillomavirus. Oncol Rep 11: 647-654, 2004.

20. Shen ZY, Xu LY, Chen MH, Cai WJ, Shen J, Chen JY and Zeng Y: Cytogenetic and molecular genetic changes in malignant transformation of immortalized esophageal epithelial cells. Int J Mol Med 12: 219-224, 2003.

21. Livak KJ and Schmittgen TD: Analysis of relative gene expression data using real-time quantitative PCR and the 2(-Delta Delta C(T)) method. Methods 25: 402-408, 2001.

22. Arantes V and Espinoza-Ríos J: Early esophageal squamous cell carcinoma management through endoscopic submucosal dissection. Rev Gastroenterol Mex 83: 259-267, 2018 (In English, Spanish).

23. Chen L, Deng H, Lu M, Xu B, Wang Q, Jiang J and Wu C: B7-H1 expression associates with tumor invasion and predicts patient's survival in human esophageal cancer. Int J Clin Exp Pathol 7: 6015-6023, 2014

24. Jenkins TK, Lopez AN, Sarosi GA, Ben-David K and Thomas RM: Preoperative enteral access is not necessary prior to multimodality treatment of esophageal cancer. Surgery 163: 770-776, 2018.

25. Nayan N, Bhattacharyya M, Jagtap VK, Kalita AK, Sunku R and Roy PS: Standard-dose versus high-dose radiotherapy with concurrent chemotherapy in esophageal cancer: A prospective randomized study. South Asian J Cancer 7: 27-30, 2018.

26. Weon JL and Potts PR: The MAGE protein family and cancer. Curr Opin Cell Biol 37: 1-8, 2015.

27. Cogdill AP, Frederick DT, Cooper ZA, Garber HR, Ferrone CR, Fiedler A, Rosenberg L, Thayer SP, Warshaw AL and Wargo JA: Targeting the MAGE A3 antigen in pancreatic cancer. Surgery 152 (3 Suppl 1): S13-S18, 2012.

28. Lin J,Lin L, Thomas DG, Greenson JK, Giordano TJ, Robinson GS, Barve RA, Weishaar FA, Taylor JM, Orringer MB and Beer DG: Melanoma-associated antigens in esophageal adenocarcinoma: Identification of novel MAGE-A10 splice variants. Clin Cancer Res 10: 5708-5716, 2004.

29. Park TS, Groh EM, Patel K, Kerkar SP, Lee CC and Rosenberg SA: Expression of MAGE-A and NY-ESO-1 in primary and metastatic cancers. J Immunother 39: 1-7, 2016. 